\title{
A Study on the Symmetry-Breaking Bifurcation Phenomenon in Transportation of Low-Strength Composites Sheets by Rolls
}

\author{
Renhe $\mathrm{Ji}^{*, \dagger}$, Baohua Chang ${ }^{*, \dagger}, \mathrm{Li}$ Wang ${ }^{*, \dagger}$ and Dong Du ${ }^{*, \dagger, \ddagger}$ \\ *Department of Mechanical Engineering, Tsinghua University \\ 30 Shuangqing Rd, Beijing, 100084, P. R. China \\ ${ }^{\dagger}$ Key Laboratory for Advanced Materials Processing Technology \\ Ministry of Education, P. R. China \\ ‡dudong@tsinghua.edu.cn
}

Received 7 November 2017

Revised 21 December 2017

Accepted 25 December 2017

Published 6 February 2018

\begin{abstract}
In the manufacturing of low-strength composites sheets, the symmetry-breaking bifurcation phenomenon may happen when the sheets are transported by rolls, which can lead to vibration and crack in the sheets. However, the mechanism and the conditions for this phenomenon has not been studied so far. In this paper, we propose a theoretical model to study the formation mechanism of the bifurcation phenomenon in a driven-idle-driven rolls system, and the critical length of the thin-sheets between rolls is obtained, above which the bifurcation will happen. Results show that the bifurcation occurs because the relationship between the lengths of the sheet between rolls and the tension caused by gravity in sheets is not monotonic, and the critical length is about 2.5 times the distance between the rolls. We also carry out experiments to verify the validity of the proposed model. This study is of great importance for the design of the multi-roll system to transport the low strength sheet materials, such as paper and composite sheets.
\end{abstract}

Keywords: Axially moving materials; catenary; nonlinear dynamics; symmetry-breaking bifurcation.

\section{Introduction}

Many novel composite materials, such as modern flame retardant composites, are sandwich panels consisting of face sheets and core sheet [Wang and Zhao, 2011]. The core sheet of a noncombustible sandwich panel is made of less than $25 \mathrm{wt} . \%$ polyacrylate and more than $75 \mathrm{wt} . \%$ inorganic powders, which makes it much safer than other sandwich panels Chen, 2011]. However, the core sheet is very fragile in

\footnotetext{
${ }^{\ddagger}$ Corresponding author.
}

This is an Open Access article published by World Scientific Publishing Company. It is distributed under the terms of the Creative Commons Attribution 4.0 (CC-BY) License. Further distribution of this work is permitted, provided the original work is properly cited. 


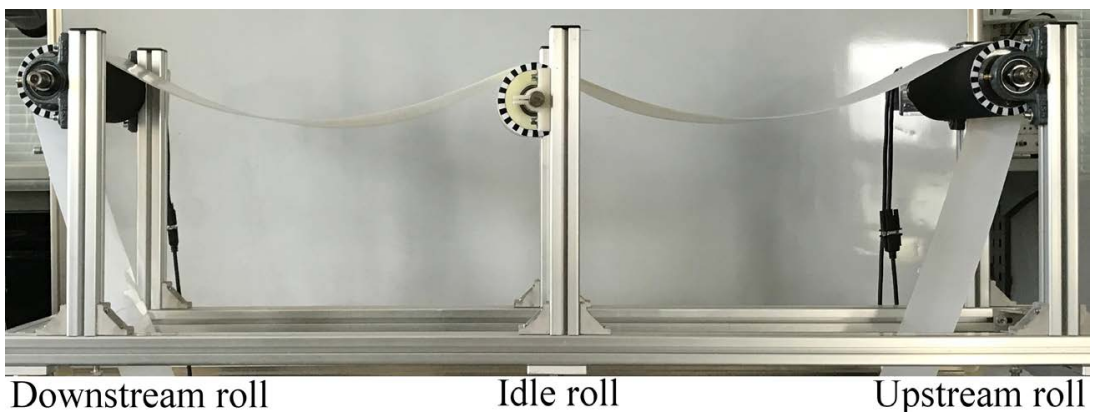

(a)

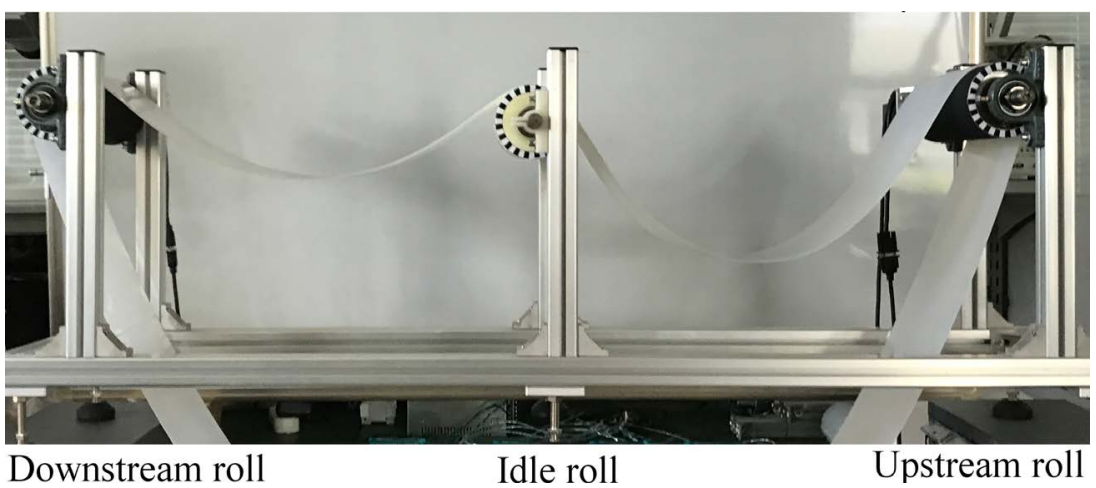

(b)

Fig. 1. (a) when the sheet length is not too long, the system can stay stable and symmetric; (b) when the sheet length is too long, the system may oscillate and stay asymmetric.

the manufacturing process and must be positioned with proper sags. We notice that when a flexible sheet is transporting through a driven-idle-driven rolls system, the system stays symmetric and stable if the length of thin-sheet material between two driven rolls is not too long. That is, the sags before and after the idle roll remain the same when the sheet moves continuously. However, when the length increases too much, the sags before and after the idle roll oscillate and finally become asymmetric. This symmetry-breaking phenomenon causes unexpected vibration in the continuous manufacture system of such low strength thin-sheet materials, as shown in Fig.1] In real manufacture system, the speed difference between driven rolls is inevitable. The speed difference may cause the length between rolls to vary, and may lead to the symmetry-breaking phenomenon, and cracking in the sheets. To avoid this phenomenon, it is necessary to reveal its causes and develop measures to tackle it.

Existing studies on the transportation of sheets by rolls system mainly focused on the high strength materials, such as cloths, in which the gravity was neglected and the sheets between rolls were assumed straight. Wickert and Mote 1990, 1991 reviewed classical vibration analysis of axially moving strings. Chen 2005]; 
Chen et al. 2009] reviewed the research on transverse vibrations and their control of axially moving strings. Frondelius et al. [2006] and Banichuk et al. [2010a studied the interaction of axially moving strings and the surrounding fluid. Laukkanen 2002 and Banichuk et al. 2010b modeled axially moving sheet as 2D plates with FEM and analytic methods, respectively, and analyzed the vibration of the sheet with proposed model. Chen et al. 2008] modeled accelerating viscoelastic strings with asymptotic approach and analyzed the steady-state response with the method. Nguven and Hong 2012] studied the influence of acceleration on the axially moving materials and its control. Alibeigloo and Liew [2015] modeled the composite beam with differential quadrature method. Recently, researchers modeled the moving sheet as Timoshenko beam [Ding et al., 2016, 2017]. Wang and Zu [2017a, 2017b] modeled the viscoelastic sheet immersed in ideal liquid and studied key parameters on the system's stability. Ghorbanpour Arani and Haghparast [2017] studied the vibration of axially moving viscoelastic microplates with Sinusoidal Shear Deformation Theory. And there are studies focused on the behavior of the moving sheet under parametric and external excitations Ding and Zu, 2013; Yan et al., 2015. Ma et al. 2017] studied the roll-to-roll system with elastic supports using analytic method. However, these models could not explain this symmetry-breaking phenomenon properly, because they all neglected the sag of the sheet and gravity. In the manufacturing of low-strength composites sheets, we notice that the symmetrybreaking bifurcation phenomenon happens when the sheets are transported by rolls, and the bifurcation phenomenon may most likely linked to the gravity. Nevertheless, this symmetry-breaking bifurcation phenomenon in the driven-idle-driven rolls system has hardly been covered by existing studies up to date.

In this study, we focus on this particular symmetry-breaking bifurcation phenomenon in the driven-idle-driven rolls system. This topic is essential to the manufacture of many novel composite materials, such as many flame retardant composites. These materials possess very low strength during manufacture $\mathrm{Su}$ et al., 2005], and this phenomenon may occur and cause unexpected vibration and defects. Also, when the sheet sag is used as feedback to control the speed of upstream and downstream rolls [Zwicky, 1957], this unique phenomenon may lead to crash of the speed control system.

This paper first proposes a model for the transportation of low strength sheets by a driven-idle-driven rolls system, in which the gravity is considered. Then, with the model developed, the formation mechanism of the symmetry-breaking bifurcation phenomenon is studied, and the critical bifurcation length of the thin sheets between the rolls is derived based on the Lyapunov stability theory. At last, experiments are carried out to verify the proposed model.

\section{Problem Formulation}

Figure 2 shows the low strength sheet transported continuously between two rolls at a constant linear velocity $v$. The upstream roll and the downstream roll are of 


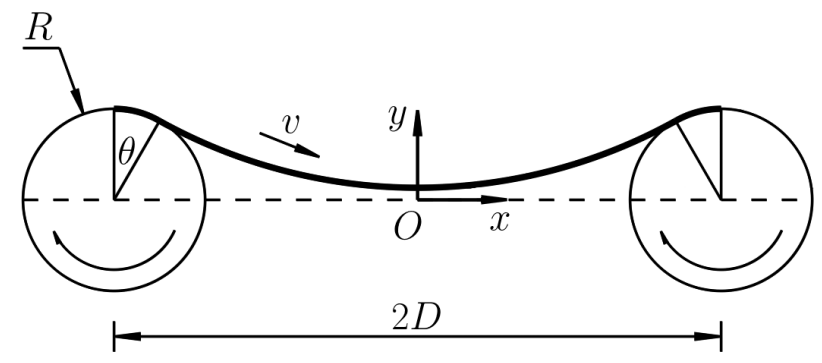

Fig. 2. The geometry of the moving sheet and rolls.

the same radius $R$ and at the same height. The distance between two rolls' axes is two-dimensional (2D). In this study, the elastic deformation in the sheet is neglected and the gravity effect is taken into consideration. Neglect the thickness of the sheet, and the linear density of the sheet is $\rho$. Touch angle $\theta$ is the center angle between the vertical direction and the touch point of the sheet and the roll. Establish a coordinate system whose origin is at the center of the axes of two rolls, $x$-axis in the horizontal direction and $y$-axis in the vertical direction.

When the two-roll system is in the stable state, the track of the sheet is a catenary [Denzler and Hinz, 1999; Stump and Fraser, 2000]. The track can be expressed as:

$$
y(x)=\frac{C_{1}}{\rho g} \cosh \left(\frac{\rho g\left(x-C_{2}\right)}{C_{1}}\right)+\frac{C_{3}}{\rho g}-\frac{v^{2}}{g} .
$$

$C_{1}, C_{2}$ and $C_{3}$ are unknown parameters and $g$ is the gravitational acceleration. The force in the sheet at position $x$ is:

$$
T(x)=\rho v^{2}+C_{1} \cosh \left(\frac{\rho g\left(x-C_{2}\right)}{C_{1}}\right) .
$$

With boundary conditions:

$$
\left\{\begin{array}{l}
y(-D+R \sin \theta)=R \cos \theta \\
y(D-R \sin \theta)=R \cos \theta \\
\left.\frac{\mathrm{d} y}{\mathrm{~d} x}\right|_{x=-D+R \sin \theta}=-\tan \theta . \\
\left.\frac{\mathrm{d} y}{\mathrm{~d} x}\right|_{x=D-R \sin \theta}=\tan \theta
\end{array}\right.
$$

$C_{1}, C_{2}$ and $C_{3}$ can be solved. Substitute $C_{1}, C_{2}$ and $C_{3}$ into Eqs. (11) and (2), the track of the sheet and the force of position $x$ can be expressed as:

$$
\begin{aligned}
y & =r_{0} \cosh \left(\frac{x}{r_{0}}\right)+R \cos \theta-\frac{r_{0}}{\cos \theta} . \\
T(x) & =\rho v^{2}+\rho g r_{0} \cosh \left(\frac{x}{r_{0}}\right)
\end{aligned}
$$


in which $r_{0}$ is:

$$
r_{0}=\frac{D-R \sin \theta}{\operatorname{arcsinh} \tan \theta} .
$$

$x \in[-D+R \sin \theta, D-R \sin \theta]$ for Eqs. (4) and (5). For a given touch angle $\theta \in[0, \pi / 2]$, the steady-state shape of the sheet and tension distribution can be determined.

With Eqs. (44) and (15), the tension in the sheet at the touch point can be derived:

$$
T_{t}(\theta)=\rho\left(v^{2}+g \frac{r_{0}}{\cos \theta}\right) .
$$

Define the sheet length between the two rolls' top point as the length between two rolls, it can be expressed as a function of $\theta$ :

$$
L(\theta)=2 \theta R+\int_{-D+R \sin \theta}^{D-R \sin \theta} \sqrt{1+\dot{y}^{2}} \mathrm{~d} x=2 \theta R+2 \tan \theta r_{0} .
$$

Define $y=0$ as the zero point of the gravitational potential energy. When the system is in the steady-state, the gravitational potential energy of the system consists of the two parts: Gravitational potential energy of the sheet on the rolls and off the rolls. The total gravitational potential energy can be given as:

$$
\begin{aligned}
U(\theta)= & \int_{-D}^{D} \rho g \sqrt{1+\dot{y}^{2}} y \mathrm{~d} x \\
= & \rho g \int_{-D+R \sin \theta}^{D-R \sin \theta}\left(r_{0} \cosh \left(\frac{x}{r_{0}}\right)+R \cos \theta-\frac{r_{0}}{\cos \theta}\right) \cdot \cosh \left(\frac{x}{r_{0}}\right) \mathrm{d} x \\
& +\rho g \int_{-D}^{-D+R \sin \theta} \sqrt{R^{2}+(x+D)^{2}} \cdot \frac{R}{\sqrt{R^{2}+(x+D)^{2}}} \mathrm{~d} x \\
& +\rho g \int_{D-R \sin \theta}^{D} \sqrt{R^{2}+(x-D)^{2}} \cdot \frac{R}{\sqrt{R^{2}+(x-D)^{2}}} \mathrm{~d} x \\
= & \left.\rho g\left[\frac{1}{2} x r_{0}+\frac{r_{0}^{2}}{4} \sinh \left(\frac{2 x}{r_{0}}\right)+\left(R \cos \theta-\frac{r_{0}}{\cos \theta}\right) r_{0} \sinh \left(\frac{x}{r_{0}}\right)\right]\right|_{-D+R \sin \theta} ^{D-R \sin \theta} \\
& +2 \rho g R^{2} \sin \theta \\
= & \rho g\left(D r_{0}+R r_{0} \sin \theta+2 R^{2} \sin \theta-r_{0}^{2} \frac{\sin \theta}{\cos ^{2} \theta}\right)
\end{aligned}
$$

A driven-idle-driven rolls system with one idle roll is shown in Fig. 3 Three rolls are of the same height and same radius, $R$. The distance between the upstream roll and the idle roll and the distance between the idle roll and the downstream roll are the same, 2D. The touch angle and potential energy of the upstream-idle roll system are $\theta_{1}$ and $U_{1}$; the touch angle and potential energy of the idle-downstream 


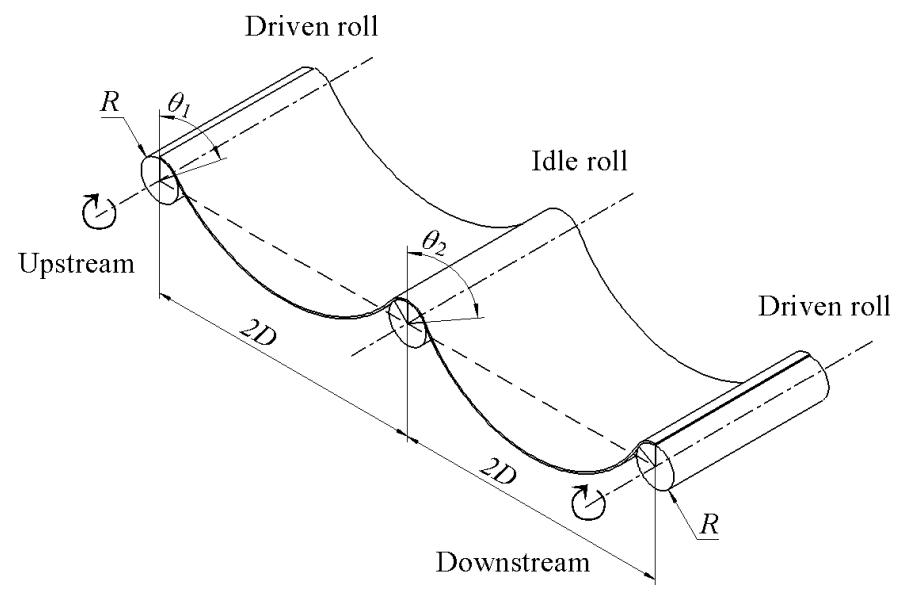

Fig. 3. The geometry of a driven-idle-driven rolls system with one idle roll.

roll system are $\theta_{2}$ and $U_{2}$. The sheet length between upstream and idle roll is $L_{1}$, and the sheet length between idle and downstream roll is $L_{2}$.

$$
U_{1}=U\left(\theta_{1}\right), \quad U_{2}=U\left(\theta_{2}\right), \quad L_{1}=L\left(\theta_{1}\right), \quad L_{2}=L\left(\theta_{2}\right) .
$$

If the system is in steady-state when the total length of sheet between upstream and downstream rolls are $2 L_{0}, \theta_{1}$ and $\theta_{2}$ should make the total potential energy minimum, e.g.,

$$
\theta_{1}, \theta_{2}=\arg \min _{\theta_{1}, \theta_{2}} U\left(\theta_{1}\right)+U\left(\theta_{2}\right) \quad \text { s.t. } L\left(\theta_{1}\right)+L\left(\theta_{2}\right)=2 L_{0} .
$$

To solve Eq. (13), introduce Lagrange function $\mathfrak{L}$ and Lagrange multiplier $\lambda$ defined as below:

$$
\mathfrak{L}\left(\theta_{1}, \theta_{2}, \lambda\right)=U\left(\theta_{1}\right)+U\left(\theta_{2}\right)-\lambda\left(L\left(\theta_{1}\right)+L\left(\theta_{2}\right)-2 L_{0}\right) .
$$

$\theta_{1}, \theta_{2}$ and $\lambda$ should satisfy:

$$
\nabla_{\theta_{1}, \theta_{2}, \lambda} \mathfrak{L}\left(\theta_{1}, \theta_{2}, \lambda\right)=0 \Rightarrow\left\{\begin{array}{l}
\frac{\partial U\left(\theta_{1}\right)}{\partial \theta_{1}}-\lambda \frac{\partial L\left(\theta_{1}\right)}{\partial \theta_{1}}=0 \\
\frac{\partial U\left(\theta_{2}\right)}{\partial \theta_{2}}-\lambda \frac{\partial L\left(\theta_{2}\right)}{\partial \theta_{2}}=0 \\
L\left(\theta_{1}\right)+L\left(\theta_{2}\right)-2 L_{0}=0
\end{array} .\right.
$$

For any given $L_{0}>2 D$, Eq. (15) can be solved using numerical methods. For example, when $R=0.05, D=0.2$ and $L_{0} \in[0.45,0.6]$, the solution for $\theta_{1}$ and $\theta_{2}$ is shown in Fig. 4 When $L_{0}$ is smaller than a certain value $\hat{L}_{0}$ (the critical length), Eq. (15) has only one solution and $\theta_{1}=\theta_{2}$; when $L_{0}>\hat{L}_{0}$, Eq. 15 has three solutions. In these three solutions, $\theta_{1}$ and $\theta_{2}$ may have the relationship $\theta_{1}>\theta_{2}, \theta_{1}=\theta_{2}$ and $\theta_{1}<\theta_{2}$. For the stable equilibrium state, the solutions are 


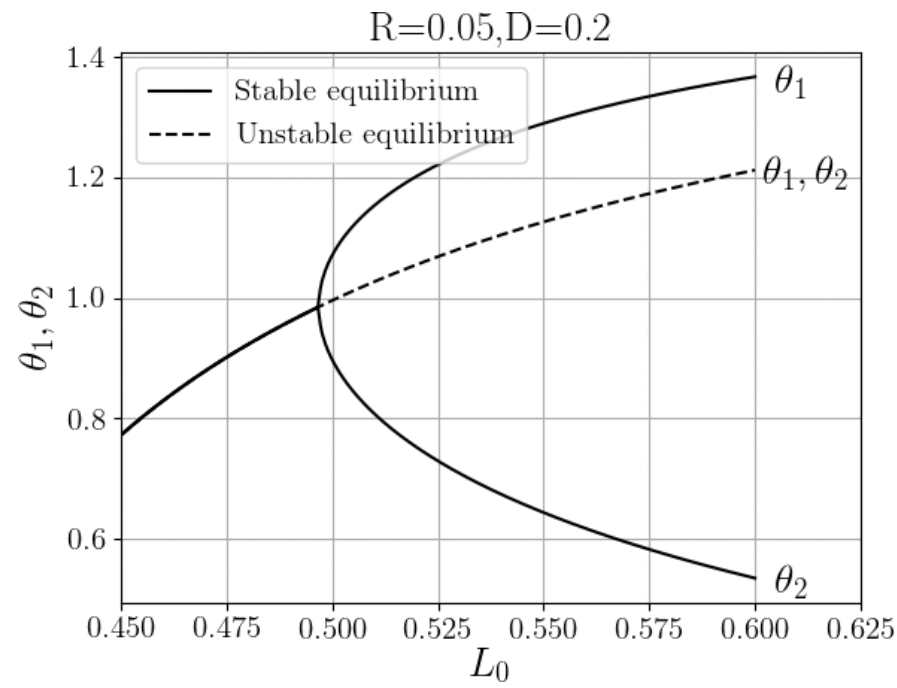

Fig. 4. Numerical solutions for Eq. (15).

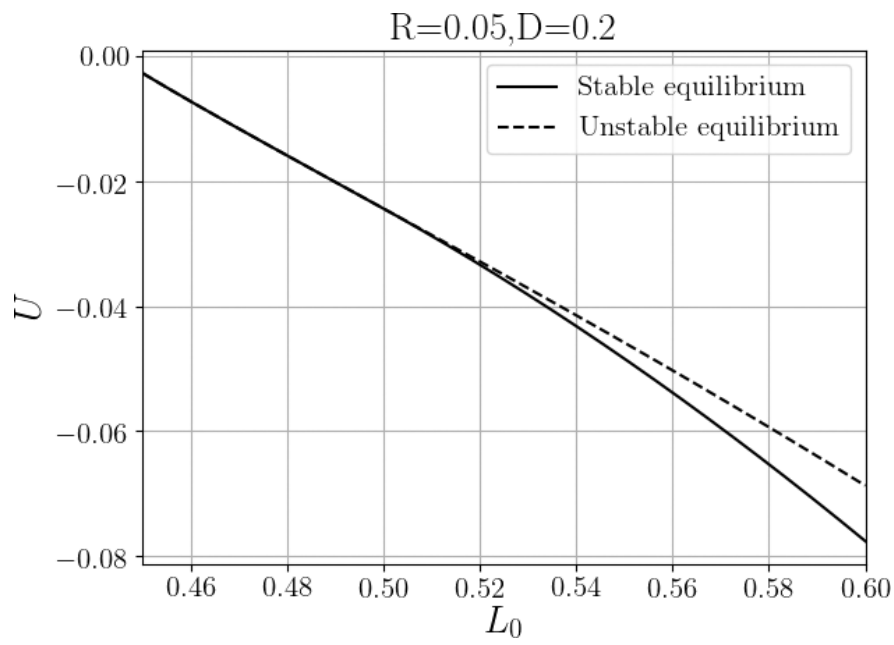

Fig. 5. Total potential energy $U_{1}+U_{2}$ versus $L_{0}$.

shown in Fig. 4 marked as the solid line; the unstable equilibrium state solutions are marked as the dashed line.

Figure 5 shows the potential energy of the system's equilibrium state when $L_{0}$ varies. The potential energy of the symmetric state is bigger than the potential energy of the asymmetric when $L_{0}>\hat{L}_{0}$. The system would be in the unstable equilibrium state when $L_{0}>\hat{L}_{0}$.

Figure 6 shows the $U-L_{1}$ curve when $L_{0}$ is smaller, equal and bigger than the critical length $\hat{L}_{0}$. The star on the curve marks the stable equilibrium point. 
R. Ji et al.
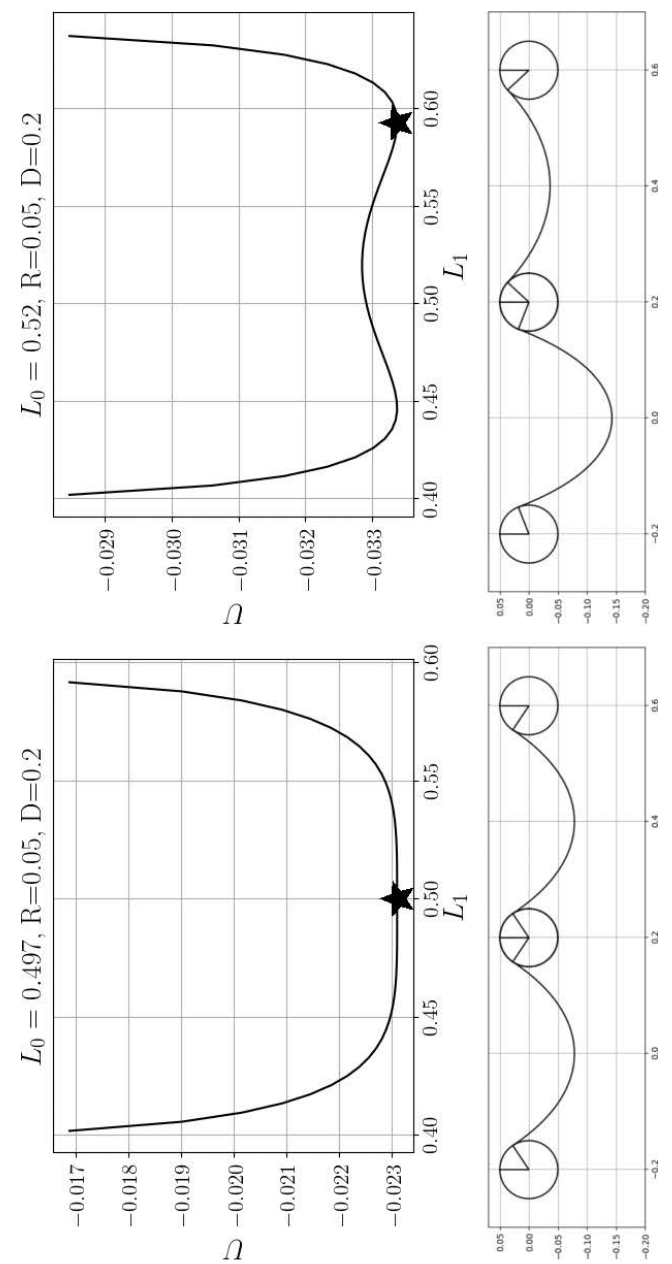

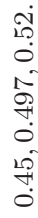

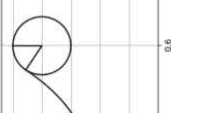

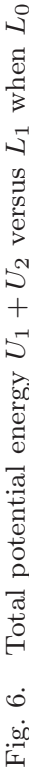
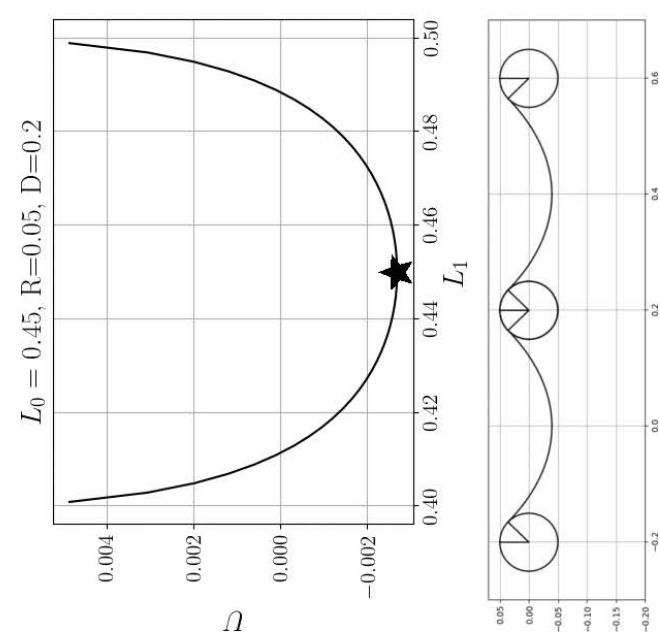
As shown in Fig. 6] when $L_{0}<\hat{L}_{0}$, the system remains stable when $L_{1}=L_{2}$; when $L_{0}>\hat{L}_{0}$, the system remains stable when $L_{1} \neq L_{2}$. As $L_{0}$ grows bigger, the system will transit from the symmetric state to the asymmetric state spontaneously.

\section{Determination of the Critical Length}

To derive an explicit expression of the critical length and explore the mechanism of the phenomenon, the problem is analyzed from the view of dynamics.

The free body diagram of the idle roll is shown in Fig. $7 T_{t 1}$ and $T_{t 2}$ are the tension in the sheet at two touch points. $\omega$ is the rotational speed of the idle roll. It is hypothesized that there is no sliding between the sheet and the rolls and that when the total length is changing, the system is in a quasistatic process, e.g., the force in the sheet can be calculated with the steady-state results.

$v_{f}$ and $v_{l}$ are the linear speeds of the upstream and downstream rolls, $v_{f} \approx v_{l}$; The moment of inertia of the idle roll is $I$. The friction coefficient of the idle roll is $\mu$. The dynamical equation of the idle roll is:

$$
\left\{\begin{array}{l}
\dot{\omega}=\frac{R}{I}\left(T_{t 2}-T_{t 1}\right)-\mu \omega \\
\dot{L}_{1}=-R \omega+v_{f} \\
\dot{L}_{2}=R \omega-v_{l}
\end{array} .\right.
$$

Manipulate Eq. (16) into a state space form, $L^{-1}(\cdot)$ denotes the inverse function of Eq. (8):

$$
\left[\begin{array}{c}
\dot{\omega} \\
\dot{L}_{1} \\
\dot{L}_{2}
\end{array}\right]=\left[\begin{array}{ccc}
-\mu & -\frac{R}{I} T_{t}\left(L^{-1}(\cdot)\right) & \frac{R}{I} T_{t}\left(L^{-1}(\cdot)\right) \\
-R & 0 & 0 \\
R & 0 & 0
\end{array}\right]\left[\begin{array}{c}
\omega \\
L_{1} \\
L_{2}
\end{array}\right]+\left[\begin{array}{c}
0 \\
v_{f} \\
-v_{l}
\end{array}\right]
$$

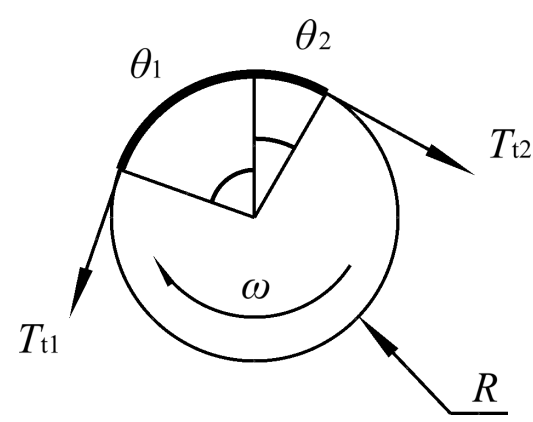

Fig. 7. Free body diagram of the idle roll. 

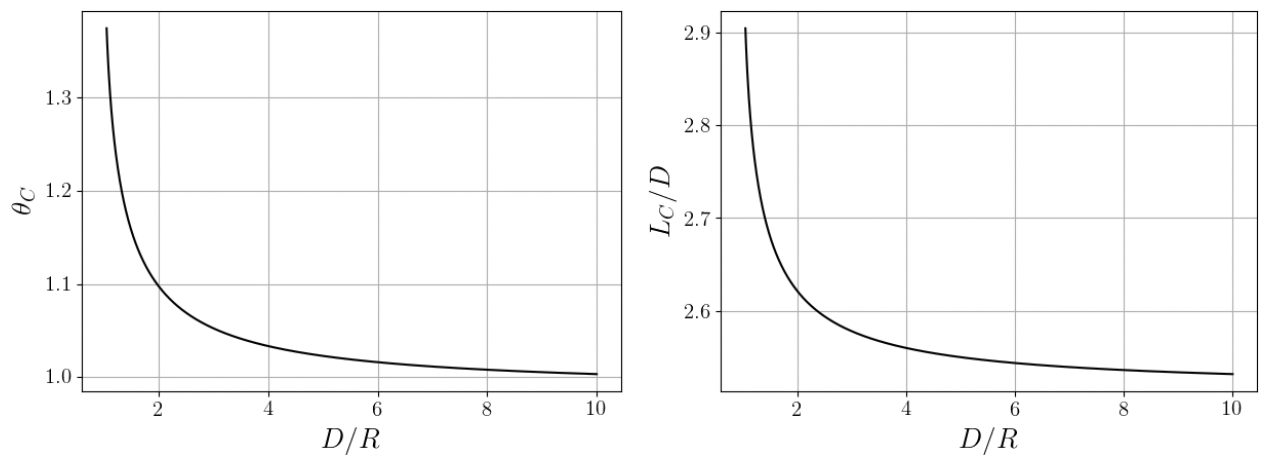

Fig. 8. $\hat{\theta}$ versus $D / R$ and $\hat{L}_{0} / D$ versus $D / R$.

When the system in the stable state near $L_{1}=L_{2}=L_{0}$, Eq. (17) can be linearized as:

$$
\left[\begin{array}{c}
\dot{\omega} \\
\dot{L}_{1}-\dot{L}_{2}
\end{array}\right]=\left[\begin{array}{cc}
-\mu & -\left.\frac{R}{I} \frac{\partial T_{t}}{\partial L}\right|_{L=L_{0}} \\
-R & 0
\end{array}\right]\left[\begin{array}{c}
\omega \\
L_{1}-L_{2}
\end{array}\right]+\left[\begin{array}{c}
0 \\
v_{f}+v_{l}
\end{array}\right]
$$

So this system being Lyapunov stable around $L_{1}-L_{2}=0$ is equivalent to:

$$
\left.\frac{\partial T_{t}}{\partial L}\right|_{L=L_{0}}<0
$$

So the critical length $\hat{L}_{0}$ will satisfy:

$$
\left.\frac{\partial T_{t}}{\partial L}\right|_{L=\hat{L}_{0}}=0
$$

Substitute Eqs. (7) and (8) into Eq. (18) and simplify the result. Equation (18) is equivalent to:

$$
(R \sin \hat{\theta}-D)+(D \sin \hat{\theta}-R) \operatorname{arcsinh} \tan \hat{\theta}=0 .
$$

$\hat{\theta}$ makes $L(\hat{\theta})=\hat{L}_{0}$. Equation 19 is solved with numerical methods. The result is shown in Fig. 8

It can be found that when $D / R \rightarrow \infty, \hat{\theta} \rightarrow 0.9855$ and $\hat{L}_{0} / D \rightarrow 2.5155$.

From Eqs. (17) and (18), it can be arrived that the reason for the bifurcation is that the $L \sim T_{t}$ curve is not linear and the oscillation may occur when $\frac{\partial T_{t}}{\partial L}=0$. The bifurcation is not relevant to the factors like friction, the transporting speed or if the rolls are of the same height.

\section{Simulation and Experiments}

To verify the proposed model, numerical simulation and experiments were conducted. Experimental parameters: sheet density $\rho=1150 \mathrm{~kg} / \mathrm{m}^{3}$, sheet thickness 


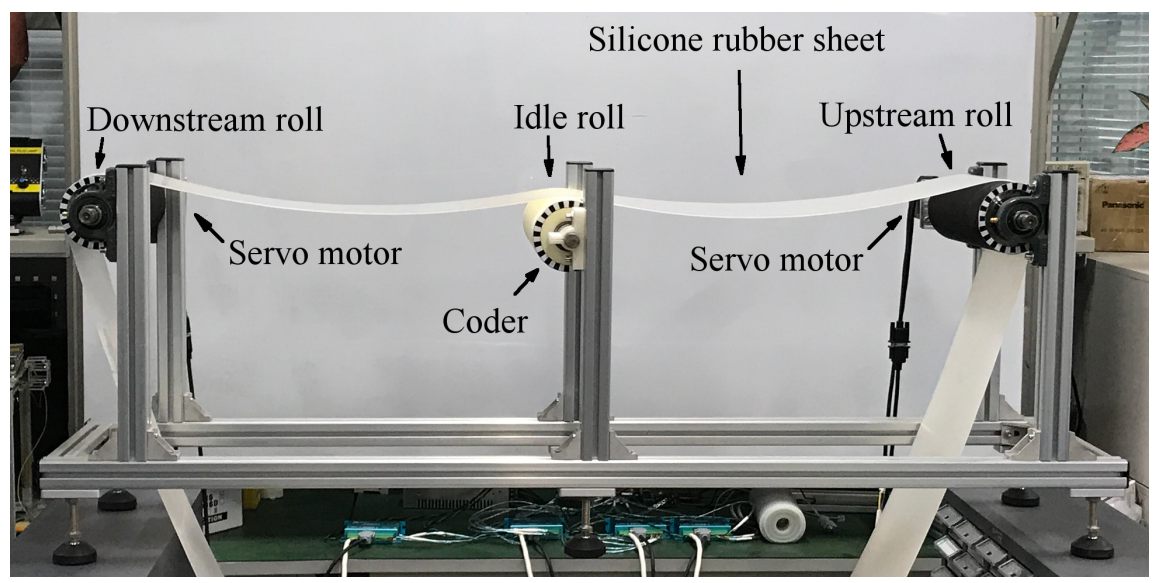

Fig. 9. Experimental set-up.

$t=0.001 \mathrm{~m}$, sheet width $d=0.2 \mathrm{~m}$, idle roll radius $R=0.05 \mathrm{~m}$, idle roll moment of inertia $I=0.00313 \mathrm{~kg} \cdot \mathrm{m}^{2}, \mu=0.05$. The sheet is made of silicone rubber. $D, v_{f}$ and $v_{l}$ are variables.

The experimental set-up is shown in Fig. 9. Two servo motors are used to drive the rolls at a constant speed. Coders are installed on the rolls. $v_{f}$ and $v_{l}$ are set to be really close and $v_{f}>v_{l}$. When the system is running, a high-speed camera is used to shoot the whole experimental set-up. The position-time curves of three rolls can be obtained by applying image processing techniques on the image sequences of three coders.

Numerical simulation (calculated with Eq. (16)) and experiment results are shown in Fig. 10. In this experiment, $2 \mathrm{D}=0.625 \mathrm{~m}, v_{f}=0.16 \mathrm{~m} / \mathrm{s}, v_{l}=0.14 \mathrm{~m} / \mathrm{s}$ and $\hat{L}_{0}=0.795 \mathrm{~m}$ according to Eqs. (19) and (8). $L_{1}$ and $L_{2}$ are calculated with the position difference between the rolls. When the system starts running, it remains stable and symmetric $\left(L_{1}=L_{2}\right)$. However, when $L_{0}$ comes near to $\hat{L}_{0}$, the system starts to oscillate around the stable point. At $L_{0}>\hat{L}_{0}$, the system becomes unstable and asymmetric. It is noticed that in both simulation and experiment, the system becomes unstable a little bit earlier than the theoretical results (the horizontal line at $\hat{L}_{0}$ ). The experiment results of bifurcation length is around $0.75 \mathrm{~m}$. This is mainly caused by the friction of the idle roll. The bigger $\mu$ is, the earlier $L_{1}$ and $L_{2}$ begin to separate according to simulation results.

Figures 11 and 12 show the simulation and experiment results when $v_{f}$ and $v_{l}$ took different values. Those figures show that transporting speed does not have much influence on $\hat{L}_{0}$.

To consolidate the conclusion that transporting speed does not have much influence on $\hat{L}_{0}$ and validate our model, another experiment is taken. In this experiment, we fix two driven rolls. At the beginning of the experiment, we fix all rolls and set the system in the symmetric steady state with the total length of the sheet between two 


\section{R. Ji et al.}

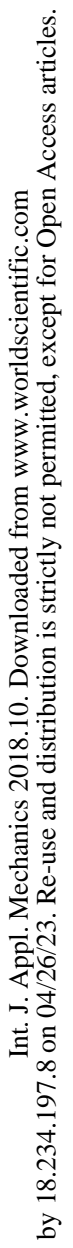
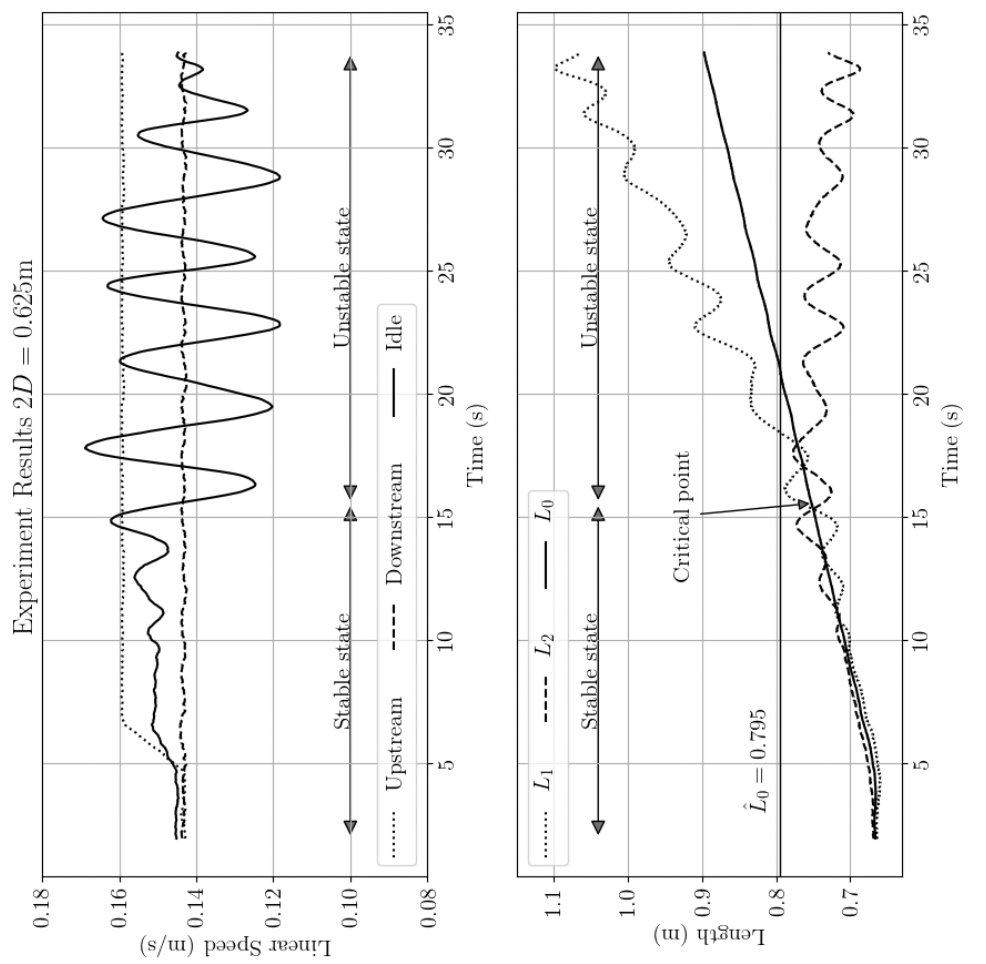

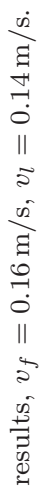
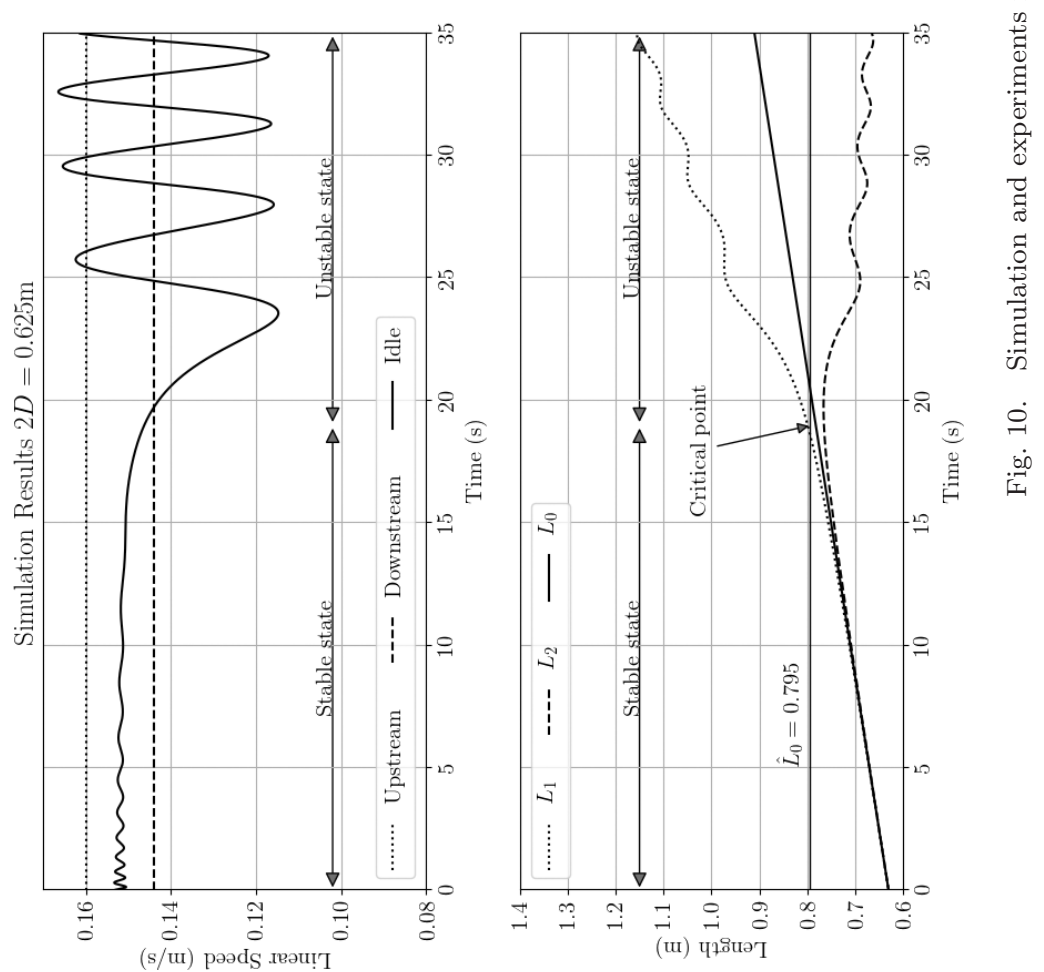
The Symmetry-Breaking Bifurcation in Transportation of Low-Strength Composites Sheets

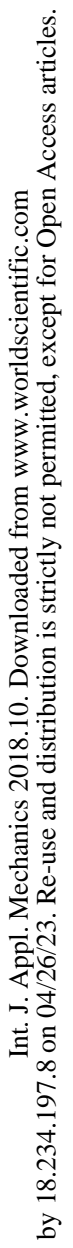
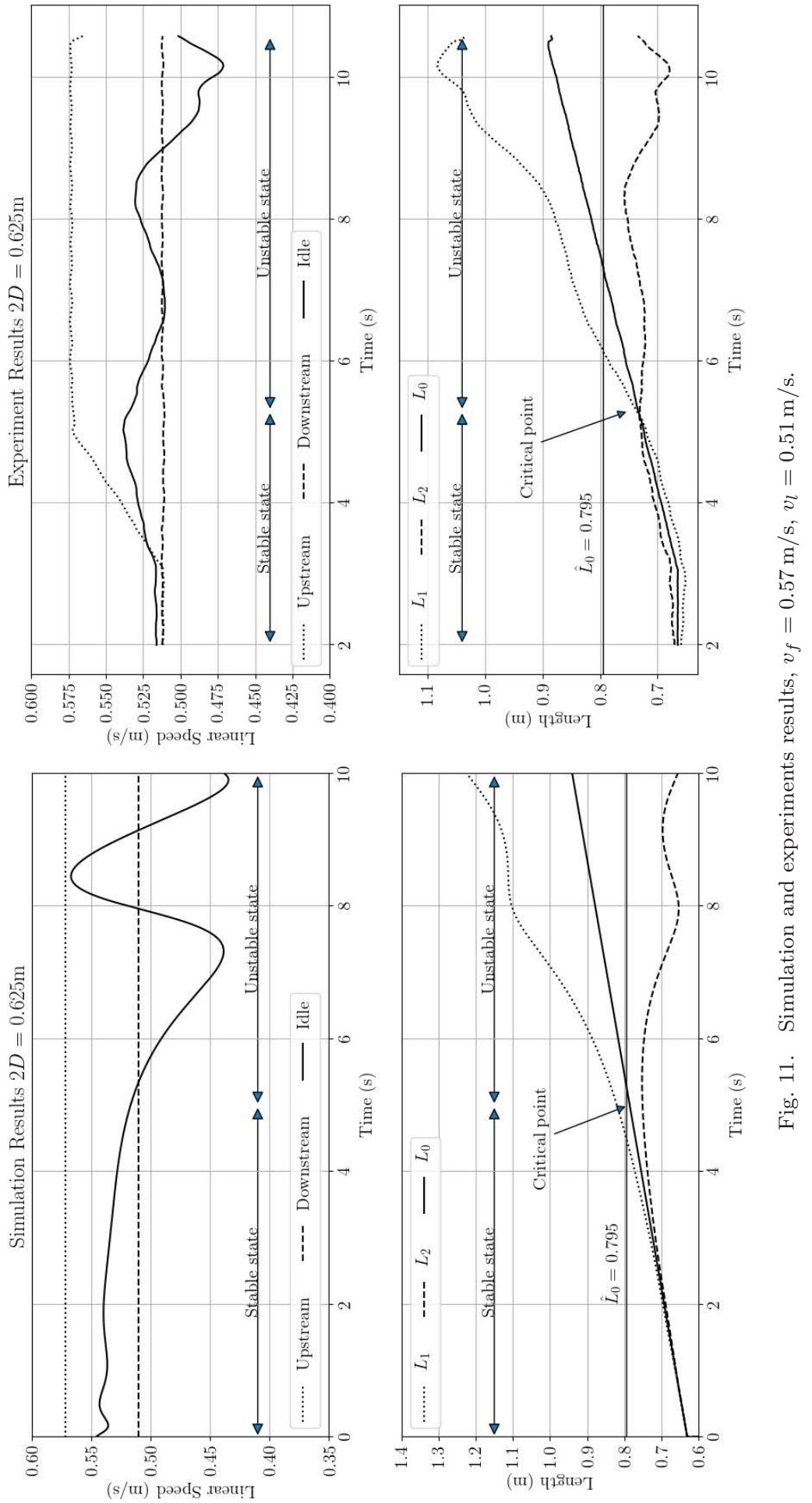
R. Ji et al.

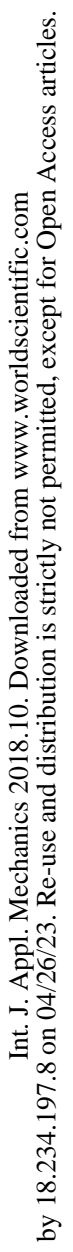
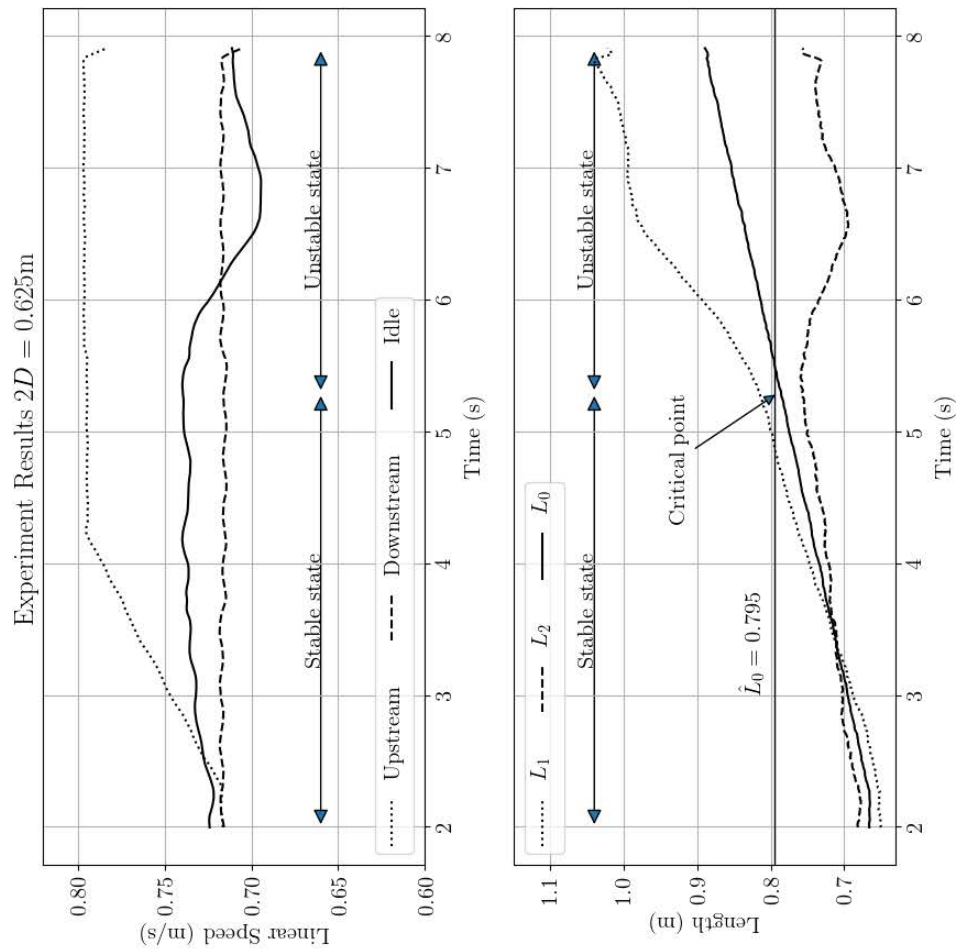

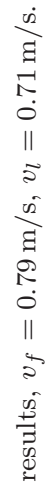
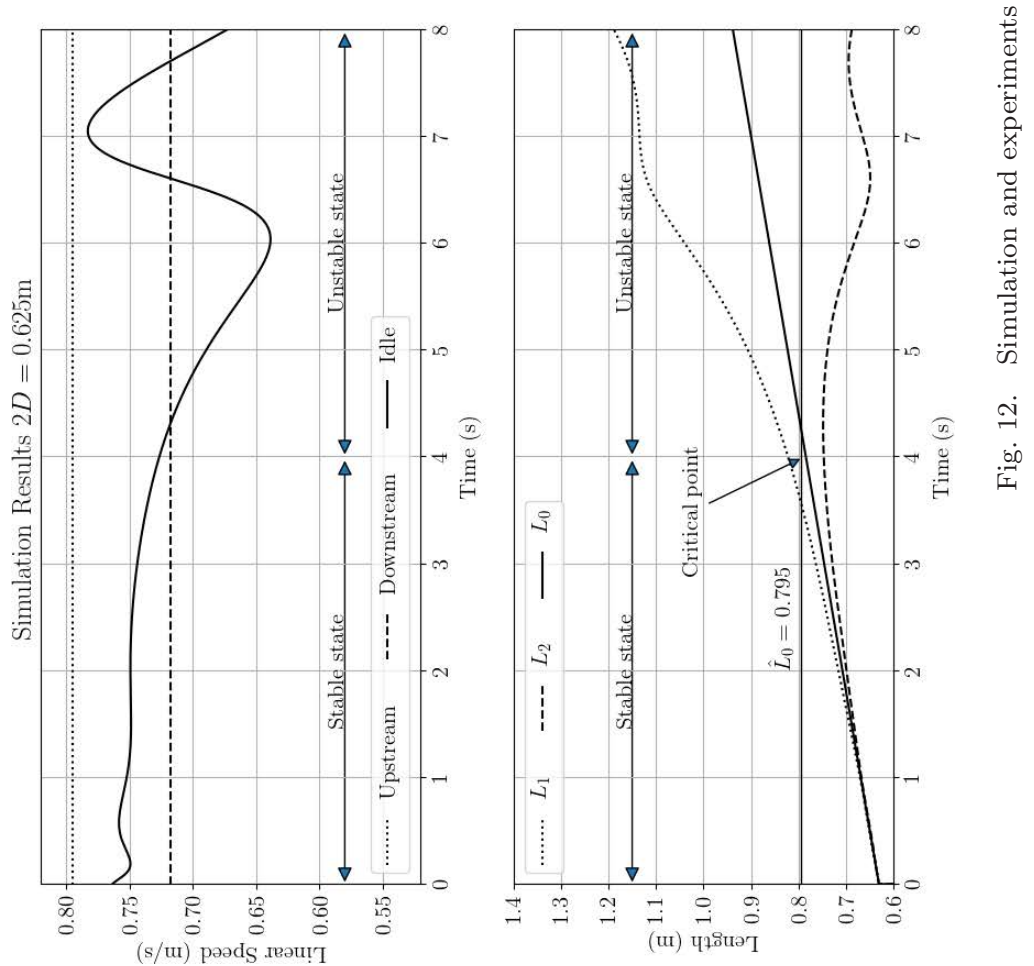

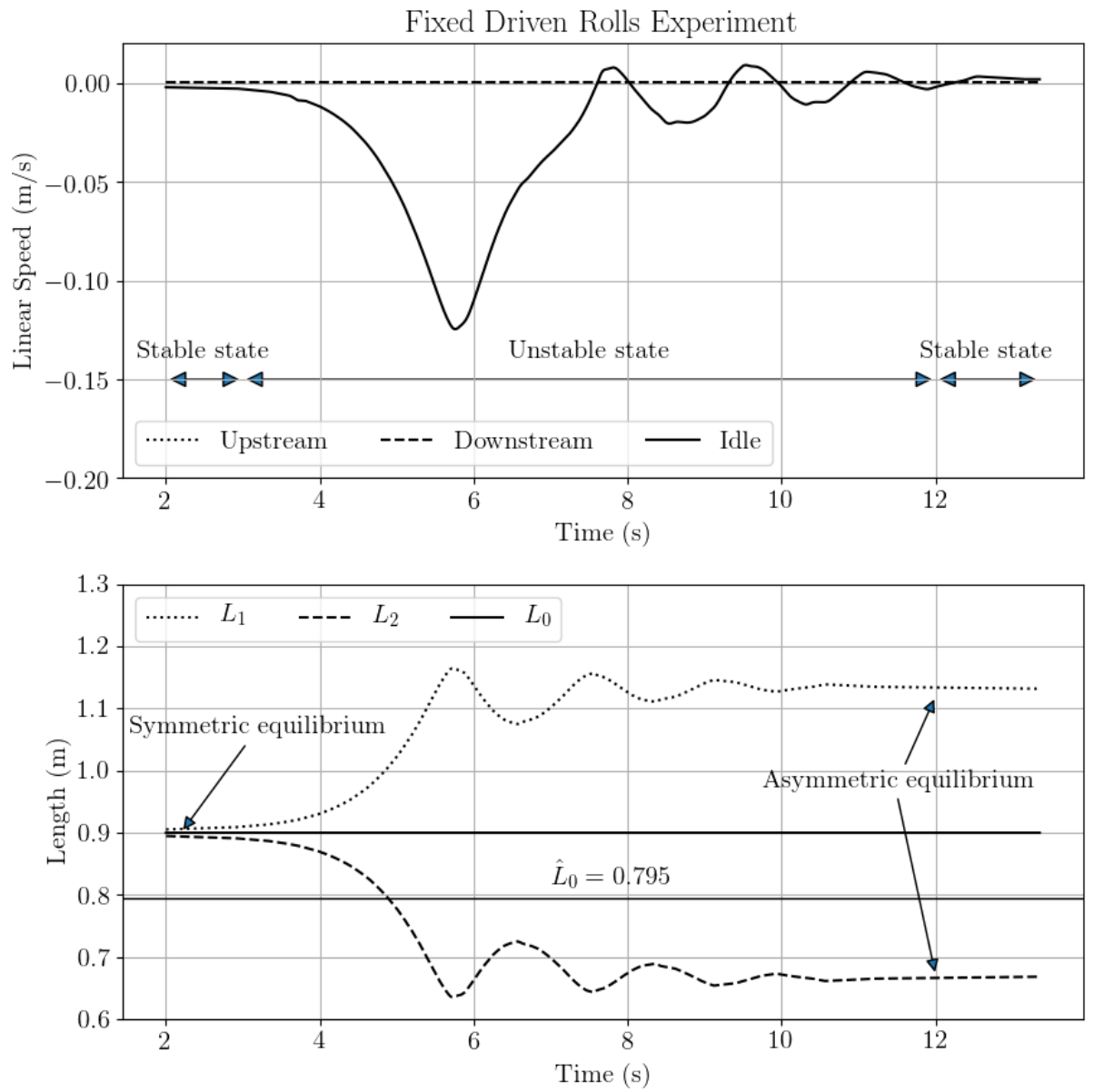

Fig. 13. Experiments results with two fixed driven rolls, $v_{f}=0 \mathrm{~m} / \mathrm{s}, v_{l}=0 \mathrm{~m} / \mathrm{s}$.

driven rolls bigger than the critical length. Then, we set the idle roll free and record the system response. As shown in Fig. 13, the system would turn to asymmetric equilibrium spontaneously.

\section{Conclusions}

The symmetry-breaking bifurcation phenomenon is studied in the transportation of the low-strength thin sheet by the driven-idle-driven rolls system, and the formation mechanisms and conditions of bifurcation are addressed. The main conclusions are drawn as follows:

(1) In the transportation of the low strength thin sheet by the driven-idle-driven rolls system, the relationship between the lengths of the sheet between rolls and the tension caused by gravity in the sheet is not monotonic, which is 
the fundamental cause of the symmetry-breaking bifurcation phenomenon happened during the sheet transportation.

(2) The transportation process may keep stable, and the bifurcation will not happen, if the total length of sheets between the upstream roll and the downstream roll is smaller than the critical length, which is about 2.5 times the distance between the upstream roll and the downstream roll. The critical length is not influenced by the transportation speeds.

\section{Acknowledgments}

This work is sponsored by China Jiangsu Science and Technology Developing Funding, No. BA2012099 and National Natural Science Foundation of China, U1537205.

\section{References}

Alibeigloo, A. and Liew, K. M. [2015] "Elasticity solution of free vibration and bending behavior of functionally graded carbon nanotube-reinforced composite beam with thin piezoelectric layers using differential quadrature method," International Journal of Applied Mechanics 07(01), 1550002.

Banichuk, N., Jeronen, J., Neittaanmki, P. and Tuovinen, T. [2010a] "On the instability of an axially moving elastic plate," International Journal of Solids and Structures 47(1), 91-99.

Banichuk, N., Jeronen, J., Neittaanmki, P. and Tuovinen, T. [2010b] "Static instability analysis for travelling membranes and plates interacting with axially moving ideal fluid," Journal of Fluids and Structures 26(2), 274-291.

Chen, J. [2011] A method to manufacture a2 class non-conbustible aluminum composite panels, CN 201010242369.5.

Chen, L.-Q. [2005] "Analysis and control of transverse vibrations of axially moving strings," Applied Mechanics Reviews 58(2), 91-116.

Chen, L.-Q., Chen, H. and Lim, C. [2008] "Asymptotic analysis of axially accelerating viscoelastic strings," International Journal of Engineering Science 46(10), 976-985.

Chen, L.-Q., Zhang, W. and Zu, J. W. [2009] "Nonlinear dynamics for transverse motion of axially moving strings," Chaos, Solitons $\&$ Fractals 40(1), 78-90.

Denzler, J. and Hinz, A. M. [1999] "Catenaria vera - the true catenary," Expositiones Mathematicae 17, 117-142.

Ding, H., Tan, X. and Dowell, E. H. [2017] "Natural frequencies of a super-critical transporting timoshenko beam," European Journal of Mechanics - A/Solids 66 (Supplement C), 79-93.

Ding, H., Tan, X., Zhang, G.-C. and Chen, L.-Q. [2016] "Equilibrium bifurcation of highspeed axially moving timoshenko beams," Acta Mechanica 227(10), 3001-3014.

Ding, H. and Zu, J. W. [2013] "Periodic and chaotic responses of an axially accelerating viscoelastic beam under two-frequency excitations," International Journal of Applied Mechanics 05(02), 1350019.

Frondelius, T., Koivurova, H. and Pramila, A. [2006] "Interaction of an axially moving band and surrounding fluid by boundary layer theory," Journal of Fluids and Structures 22(8), 1047-1056. 
Ghorbanpour Arani, A. and Haghparast, E. [2017] "Size-dependent vibration of axially moving viscoelastic micro-plates based on sinusoidal shear deformation theory," International Journal of Applied Mechanics 09(02), 1750026.

Laukkanen, J. [2002] "Fem analysis of a travelling web," Computers ES Structures 80(24), $1827-1842$.

Ma, L., Chen, J., Tang, W. and Yin, Z. [2017] "Free vibration analysis of an axially travelling web with intermediate elastic supports," International Journal of Applied Mechanics 09(07), 1750104.

Nguyen, Q. C. and Hong, K.-S. [2012] "Simultaneous control of longitudinal and transverse vibrations of an axially moving string with velocity tracking," Journal of Sound and Vibration 331(13), 3006-3019.

Stump, D. and Fraser, W. [2000] "Bending boundary layers in a moving strip," Nonlinear Dynamics 21(1), 55-70.

Su, Z., Jiang, P., Li, Q., Wei, P. and Zhang, Y. [2005] "Toughening of polypropylene highly filled with aluminum hydroxide," Polymers \& Polymer Composites 13(2), 139-150.

Wang, X. and Zhao, C. [2011] "Research on the fireproof safety test and material application of the halogen-free high flame retardant aluminium-plastic composite panels used for exterior wall decoration of high-rise buildings," China Building Materials Science $\& 3$ Technology 30(6), 14-24.

Wang, Y. Q. and Zu, J. W. [2017a] "Instability of viscoelastic plates with longitudinally variable speed and immersed in ideal liquid," International Journal of Applied Mechanics 09(01), 1750005.

Wang, Y. Q. and Zu, J. W. [2017b] "Nonlinear dynamics of functionally graded material plates under dynamic liquid load and with longitudinal speed, International Journal of Applied Mechanics 09(04), 1750054.

Wickert, J. A. and Mote, C. D. [1990] "Classical vibration analysis of axially moving continua," Journal of Applied Mechanics 57(3), 738-744.

Wickert, J. A. and Mote, C. D. [1991] "Response and discretization methods for axially moving materials," Applied Mechanics Reviews 44(11S), S279-S284.

Yan, Q., Ding, H. and Chen, L. [2015] Nonlinear dynamics of axially moving viscoelastic timoshenko beam under parametric and external excitations," Applied Mathematics E Mechanics 36(8), 971-984.

Zwicky, R. [1957] "Automation in continuous operating rolling-mill trains," Elektrotechnische Zeitschrift. Ausgabe A: Zentralblatt fur Elektrotechnik 78(23), 869-873. 